TOKYO J. MATH.

VoL. 5, No. 1, 1982

\title{
A Construction of the Groups of Units of Some Number Fields from Certain Subgroups
}

\author{
Ken NAKAMULA \\ Tokyo Metropolitan University
}

\section{Introduction}

All number fields we consider are in the complex number field. The symbol $\langle S\rangle$ denotes a multiplicative group generated by an element or a set $S$. For any complex number $x$, a $j$-th root of $x$, which is taken to be positive real if $x$ is positive real, is denoted by $\sqrt[3]{x}$.

0.1. For a finite algebraic number field $k$, let $E_{k}$ be the group of units of $k$ and $W_{k}$ be the torsion part of $E_{k}$. Then $E_{k}$ is generated by $W_{k}$ and by a set $\left\{\varepsilon_{j} \mid j=1, \cdots, r\right\}$ of fundamental units of $k$. The number $r$ is called the Dirichlet number of $k$. In general, some geometrical calculation is necessary to obtain fundamental units of $k$ (see [1] or Chap. 2 , $\$ 5.3$ of [2]). Those methods are very complicated when $r$ is large. If $k$ is a real abelian number field, there is an effective method, which requires no geometrical calculation, to obtain fundamental units of $k$ (see [5]). Our main interest is, in case $k$ is not galois or galois but not abelian over $\boldsymbol{Q}$, to construct $\left\{\varepsilon_{j} \mid j=1, \cdots, r\right\}$ from certain subgroups of $E_{k}$ without any geometrical calculation. Let $E_{k}^{\prime}$ be the subgroup of $E_{k}$ generated by $W_{k}$ and the units of all proper subfields of $k$. If the index $\left(E_{k}: E_{k}^{\prime}\right)$ is finite, we may construct $E_{k}$ from $E_{k}^{\prime}$. Such a problem is treated in some cases when $k$ is galois over $Q$, see [6] and [7] for example. If $\left(E_{k}: E_{k}^{\prime}\right)$ is not finite, we consider the following subgroup $H_{k}$, the group of relative units of $k$, in addition to $E_{k}^{\prime}$ :

$$
H_{k}=\left\{\varepsilon \in E_{k} \mid N_{k / k_{1}}(\varepsilon) \in W_{k} \text { for any proper subfield } k_{1} \text { of } k\right\} \text {. }
$$

The object of the present article is to show a way how $E_{k}$ is constructed from $E_{k}^{\prime}$ and $H_{k}$ in some cases. Our main tool is Proposition 1 in $\S 1$, which can be applied to $k$ of types as in 1.2. To explain our actual calculation, we take for $k$ a subfield of a dihedral extension of 
degree 8 or 12 over $Q$. Most of the results have been announced in our previous note [12] without proof.

0.2. Throughout the paper we suppose $n=2$ or 3 . Let $L$ be a dihedral extension of degree $4 n$ over $Q$ with the galois group $G$ :

$$
G=\langle\sigma, \tau\rangle ; \quad \sigma^{2 n}=\tau^{2}=(\sigma \tau)^{2}=1 .
$$

Let $K, F$ and $\Omega$ be the invariant subfield of $\langle\tau\rangle,\left\langle\sigma^{3} \tau\right\rangle$ and $\left\langle\sigma^{n}\right\rangle$ respectively. The quadratic subfields $K_{2}$ and $F_{2}$ of $K$ and $F$ are the invariant subfields of $\left\langle\sigma^{2}, \tau\right\rangle$ and $\left\langle\sigma^{3} \tau, \sigma^{2}\right\rangle$ respectively. When $n=3$, the cubic subfield $K_{3}=K \cap F$ is the invariant subfield of $\left\langle\sigma^{3}, \tau\right\rangle$. The quartic subfield $\Lambda=K_{2} \cdot F_{2}$ is the invariant subfield of $\left\langle\sigma^{2}\right\rangle$ and is the maximal abelian subfield of $L$. Another quadratic subfield $\Lambda_{2}$, which is the invariant subfield of $\langle\sigma\rangle$, is contained in $\Lambda$. Note that $\Lambda=\Omega$ when $n=2$, and that $W_{L}=W_{A}$ in any case. We shall investigate the groups $E_{R}, E_{F}$ and $E_{L}$. The fields $K$ and $F$ are pure number fields of degree $2 n$ if and only if $\Lambda_{2}$ is the $2 n$-th cyclotomic field, and then

$$
K=Q(\sqrt[2 n]{d}) \text { and } F=Q\left(\sqrt[2]{-n^{n} d}\right)
$$

with a natural number $d$.

T. Nagell [10], in case $n=2$, and H.-J. Stender [16], in case $n=3$ and $K$ is pure, have given a classification of $K$ and $F$ in terms of the structure of $E_{K} / E_{K}^{\prime} \cdot H_{K}$ and $E_{F} / E_{F}^{\prime} \cdot H_{F}$. We can complete such a classification as a corollary of Proposition 1. Moreover, Proposition 1 has another application to the field of type $Q(\sqrt[p]{f}, \sqrt[p]{g})$ as in Proposition 4 in 1.5 .

0.3. Let $k / Q$ be a finite galois extension with the galois group $G^{\prime}$. A. Brumer [4], L. Bouvier-J. Payan [3] and N. Moser [8] have investigated the structure of $E_{k}$ as a $Z\left[G^{\prime}\right]$-module when $G^{\prime}$ is a certain cyclic or dihedral group, and have given several conditions for $k$ to have a Minkowski unit (a unit which forms, together with some of its conjugates, a set of fundamental units of $k$ ). But in our case when $k=L$ and $G^{\prime}=G$ given as above, there seems to be no literature concerning Minkowski units. Let us assume $L \cap R=K$. Then, by the result in $\S 1$, we see

$$
\left.H_{K}=\langle-1\rangle \times\left\langle\varepsilon_{1}\right\rangle \text { with } \varepsilon_{1}\right\rangle 1 .
$$

Let $\eta_{2}(>1)$ be the fundamental unit of $K_{2}$. Further let $\eta_{3}(>1)$ be the fundamental unit of $K_{3}$ when $n=3$. Then we can prove the following 
theorem by considering the relation between $E_{K}$ and $E_{F}$ and by constructing $E_{L}$ from $E_{L}^{\prime}$.

THEOREM 1. Let assumptions be as above. (i) In case $n=2$, there is a real Minkowski unit for $L$ if and only if the following holds:

$$
\begin{aligned}
& E_{K}=H_{K} \times\left\langle\varepsilon_{2}\right\rangle \text { with } \varepsilon_{2}=\sqrt{\varepsilon_{1} \eta_{2}}, \\
& E_{4}=E_{A}^{\prime} \text { and } K \neq K_{2}\left(\sqrt{2 \eta_{2}}\right), \quad \neq Q(\sqrt[4]{2}) .
\end{aligned}
$$

Moreover, if this condition is satisfied, the unit $\varepsilon_{2}$ is a Minkowski unit for $L$. (ii) In case $n=3$, there is a real Minkowski unit for $L$ if and only if the following holds:

$$
\begin{aligned}
& E_{K}=H_{K} \times\left\langle\varepsilon_{2}\right\rangle \times\left\langle\varepsilon_{3}\right\rangle \text { with } \varepsilon_{2}=\sqrt[8]{\varepsilon_{1} \eta_{2}^{ \pm 1}}, \quad \varepsilon_{3}=\sqrt{\varepsilon_{1} \eta_{3}}, \\
& E_{\Omega}=E_{0}^{\prime} \text { and } E_{A}=E_{\Lambda}^{\prime} .
\end{aligned}
$$

Moreover, if this condition is satisfied, the unit $\varepsilon_{2}^{-1} \varepsilon_{3}$ is a Minkowski unit for $L$.

0.4. Stender [15], [16] and [17] have given a series of $K$ with explicit fundamental units when $K$ is a pure number field. The method used there is to construct $E_{K}$ from $H_{K}$ and $E_{K}^{\prime}$. By the same method, we obtain a new series of $K$ with explicit fundamental units.

THEOREM 2. Let $d$ be a square free integer greater than 1 and put $\theta=\sqrt[2 n]{d}$. Assume $K=Q(\theta)$ has a binomial unit $a-b \theta(a, b \in N)$ such that

$$
a \geqq b^{2 n}-1 \text {. }
$$

Then a set of fundamental units of $K$ is given as follows:

$$
\begin{array}{lll}
\xi_{1}=a-b \theta, & \xi_{2}=a+b \theta & \text { in case } n=2 ; \\
\xi_{1}=a-b \theta, & \xi_{2}=a+b \theta, \quad \xi_{3}=a^{2}+a b \theta+b^{2} \theta^{2} & \text { in case } n=3 .
\end{array}
$$

Combining this theorem with Theorem 1, we obtain examples of $L$ with explicit real Minkowski units.

THEOREM 3. Assumptions being the same as in Theorem 2, the unit $\xi_{1}$ is a real Minkowski unit for the galois closure $L$ of $K / Q$ unless $d=2$.

We also give examples of $L$ with no Minkowski unit in case $n=2$, see Propositions 7 and 8.

0.5. In $\S 1$, we prove Proposion 1 and apply it to the groups of units of some number fields, especially to $E_{K}$ and $E_{F}$. (In order to 
construct $E_{x}$ and $E_{p}$, it is the most important to find a finite index subgroup of $H_{K}$ or $H_{F}$. When $L \cap R=K$, we can compute $\varepsilon_{1}$ in (1) from a so called "elliptic unit", and then we have an effective method to compute fundamental units and the class numbers of $K$ and $F$, see [13] and [14].) In $\S 2$, we study the relation between $E_{K}$ and $E_{F}$. The proof of Theorem 1 is given in $\S 3$ and the proofs of Theorems 2 and 3 are given in $\S 4$.

\section{§1. A property of a free abelian group.}

1.1. Let $E$ be a free abelian group with finite rank $r$, and $E_{i}$ be subgroups of $E$ with rank $r_{i}(1 \leqq i \leqq m)$. Assume that natural numbers $n_{i}$ and homomorphisms $f_{i}: E \rightarrow E_{\imath}(1 \leqq i \leqq m)$ are given and satisfy

$$
f_{i}(x)=x^{\delta_{i j} n_{i}} \text { for } x \in E_{j}(1 \leqq i, j \leqq m),
$$

where $\delta_{i j}$ is Kronecker's delta. Put

$$
E_{0}=\bigcap_{i=1} \operatorname{Ker}\left(f_{i}\right)
$$

and let $r_{0}$ be the rank of $E_{0}$.

Proposition 1 (Lemma 1 of [12]). Notations being as above, the following holds:

(i ) $\left\langle E_{i} \mid 0 \leqq i \leqq m\right\rangle=E_{0} \times E_{1} \times \cdots \times E_{m}$ (direct product);

(ii) The product map $f=f_{1} \times \cdots \times f_{m}: E \rightarrow E_{1} \times \cdots \times E_{m}$ induces the isomorphism

$$
E /\left(E_{0} \times \cdots \times E_{m}\right) \cong f(E) /\left(E_{1}^{n_{1}} \times \cdots \times E_{m}^{n_{m}}\right),
$$

and thus

$$
r=r_{0}+\cdots+r_{m}, \quad\left(E: E_{0} \times \cdots \times E_{m}\right) \mid n_{1}^{r_{1}} \cdots n_{m}^{r_{m}} ;
$$

(iii) If $x_{i}\left(1 \leqq i \leqq r-r_{0}\right)$ are elements of $E$ such that $f(E)=$ $\left\langle f\left(x_{i}\right) \mid 1 \leqq i \leqq r-r_{0}\right\rangle$, we have

$$
E=E_{0} \times\left\langle x_{1}\right\rangle \times \cdots \times\left\langle x_{m}\right\rangle \quad \text { (direct product); }
$$

(iv) If $n_{i}(1 \leqq i \leqq m)$ are pairwise relatively prime,

$$
f(E)=f_{1}(E) \times \cdots \times f_{m}(E) \quad \text { (direct product) . }
$$

Proof. For $i=0,1, \cdots, m-1$, let

$$
x_{i}=x_{i+1} \cdots x_{m} \text { with } x_{j} \in E_{j}(i \leqq j \leqq m) .
$$


If $i \geqq 1$, then

$$
x_{i}^{n_{i}}=f_{i}\left(x_{i}\right)=f_{i}\left(x_{i+1}\right) \cdots f_{i}\left(x_{m}\right)=1,
$$

hence $x_{i}=1$. If $i=0$, then, for $j=1, \cdots, m$,

$$
1=f_{j}\left(x_{0}\right)=f_{j}\left(x_{1}\right) \cdots f_{j}\left(x_{m}\right)=x_{j}^{n j},
$$

hence $x_{j}=1$, and so $x_{0}=1$. Therefore $E_{i} \cap\left(E_{i+1} \cdots E_{m}\right)=1$ for $i=0,1, \cdots$, $m-1$. Thus (i) is proved. To see (ii), it is enough to prove

$$
f^{-1}\left(E_{1}^{n_{1}} \times \cdots \times E_{m}^{n_{m}}\right)=E_{0} \times \cdots \times E_{m} .
$$

The right side of (2) is obviously contained in the left side. Let $x$ be an element of the left side of $(2)$, then $f_{i}(x)=x_{i}^{n_{i}}$ with $x_{i} \in E_{i}(1 \leqq i \leqq m)$, so $x\left(x_{1} \cdots x_{m}\right)^{-1} \in \operatorname{Ker}(f)=E_{0}$. Hence (2) is proved. It is easy to see (iii) from the exact sequence

$$
1 \longrightarrow E_{0} \stackrel{\text { inc. }}{\longrightarrow} E \stackrel{f}{\longrightarrow} f(E) \longrightarrow 1
$$

which splits via a natural homomorphism from $f(E)$ to $E$. Let $n_{1}, \cdots, n_{m}$ be pairwise relatively prime. Since $f(E)$ is always contained in $f_{1}(E) \cdots f_{m}(E)$, it is enough to show

$$
f_{i}(E) \subset f(E) \quad(1 \leqq i \leqq m)
$$

in order to prove (iv). Take $\mu, \nu \in Z$ such that

$$
\mu n_{1}+\nu a=1, \quad a:=n_{2} \cdots n_{m} .
$$

For $x \in E$, let

$$
y:=x^{\nu a} f_{1}(x)^{\mu}\left(\prod_{i=2}^{m} f_{i}(x)^{a_{i}}\right)^{-\nu}, \quad a_{i}:=a / n_{i},
$$

then $f(y)=f_{1}(x)$, hence $f_{1}(E) \subset f(E)$. Similarly, (3) holds for $2 \leqq i \leqq m$. Thus we complete the proof of Proposition 1.

1.2. We can apply Proposition 1 to the following finite algebraic number field $k$;

A. the field $k$ is an extension of relative degree $n_{1}$ over a proper subfield $k_{1}$ of $k$;

B. the field $k$ is the composite of two subfields $k_{1}$ and $k_{2}$, which are linearly disjoint over $Q$, with $\left[k: k_{i}\right]=n_{i}(i=1,2)$;

C. the field $k$ contains $M$ subfields $k_{i}(1 \leqq i \leqq M)$ which are pairwise linearly disjoint over $\boldsymbol{Q}$ and $\left[k: k_{i}\right]=\left[k_{i}: Q\right]=N(1 \leqq i \leqq M)$. 
In all these cases, let $E:=E_{k} / W_{k}, E_{i}:=E_{k_{i}} \cdot W_{k} / W_{k}\left(\simeq E_{k_{i}} / W_{k_{i}}\right)$ and $f_{i}:=N_{k / k_{i}}$. Then $f_{i}\left(W_{k}\right) \subset W_{k_{i}} \subset W_{k}$, so $f_{i}$ is regarded as a homomorphism from $E$ to $E_{i}$. Let $r$ and $r_{i}$ be the Dirichlet numbers of $k$ and $k_{i}$ respectively. Then the assumptions of Proposition 1 are satisfied with

$$
\begin{array}{ll}
m=1 & \text { in case A, } \\
m=2 & \text { in case B, } \\
m=M & \text { in case C. }
\end{array}
$$

Define the groups of relative units with respect to $k / k_{i}$ by

$$
H_{i}:=\left\{\varepsilon \in E_{k} \mid N_{k / k_{i}}(\varepsilon) \in W_{k_{i}}\right\} \text {. }
$$

Then $W_{k}$ is contained in $H_{i}$, and

$$
E_{0}=\left(\bigcap_{i=1}^{\infty} H_{i}\right) / W_{k} .
$$

In the rest of this section, typical examples of each case are studied.

1.3. Notations being as in 0.2 , let $n=2$. The fields $K$ and $F$ belong to the case $A$ of 1.2. Note that $E_{K}^{\prime}=E_{K_{2}}$ and $E_{F}^{\prime}=E_{F_{2}}$, because $W_{K}=W_{K_{2}}$ and $W_{F}=W_{F_{2}}$.

Assume $L$ is imaginary and the maximal real subfield of $L$ is galois over $\boldsymbol{Q}$, i.e.,

$$
L \cap R=\Omega \text {. }
$$

Then $E_{K}$ and $E_{F}$ have the same situation, so we may treat $E_{K}$. Applying Proposition 1 as in the case $A$ of 1.2 , we see $\left(E_{K}: E_{K_{2}}\right)=1$ or 2 . More precisely, we have the following.

Proposition 2 (Nagell). If $n=2$ and $L \cap R=\Omega$, then $E_{K}=E_{K_{2}}$.

Proof. See $\S 2.11$ of $[10]$, p. 359 .

Assume $L$ is imaginary and the maximal real subfield of $L$ is not galois over $Q$. Then we may assume

$$
L \cap R=K \text {. }
$$

In this case, we have

$$
E_{K_{2}}=\langle-1\rangle \times\left\langle\eta_{2}\right\rangle \quad \text { with } \quad \eta_{2}>1, \quad E_{F_{2}}=W_{F_{2}} .
$$

Applying Proposition 1 as in the case $A$ of 1.2 , we see 
(5) $\quad H_{K}=\langle-1\rangle \times\left\langle\varepsilon_{1}\right\rangle$ with $\varepsilon_{1}>1, \quad E_{F}=H_{F}=W_{F_{2}} \times\left\langle\varepsilon_{0}\right\rangle$,

and

$$
E_{R}=H_{K} \times\left\langle\varepsilon_{2}\right\rangle,
$$

where $\varepsilon_{2}$ can be chosen as one and only one of the following forms:

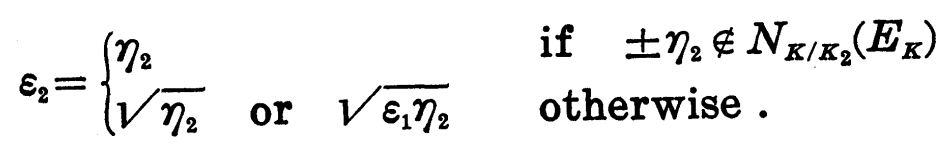

REMARK 1. Since $N_{K / K_{2}}(\alpha)^{o}=\left|\alpha^{o}\right|^{2} \geqq 0$ for $\alpha \in K$, it is obtained that

$$
H_{K}=\left\{\varepsilon \in E_{K} \mid N_{K / K_{2}}(\varepsilon)=1\right\}
$$

and that

$$
\pm \eta_{2} \notin N_{K / K_{2}}\left(E_{K}\right) \Longleftrightarrow \eta_{2}^{\sigma} \notin N_{K / K_{2}}\left(E_{K}\right)
$$

If $\varepsilon_{2}=\sqrt{\eta_{2}}$ in (7), then $K=K_{2}\left(\sqrt{\eta_{2}}\right)$, and so $\eta_{2}^{o}<0$, thus

$$
\varepsilon_{2}=\sqrt{\eta_{2}} \text { in }(7) \Longrightarrow N_{K_{2} / e}\left(\eta_{2}\right)=-1 \text {. }
$$

Assume $L$ is real, then we may treat only $E_{K}$. Similarly as above we have

$$
E_{K_{2}}=\langle-1\rangle \times\left\langle\eta_{2}\right\rangle \text { with } \eta_{2}>1
$$

and see

$$
\begin{gathered}
H_{K}=\langle-1\rangle \times\left\langle\varepsilon_{0}\right\rangle \times\left\langle\varepsilon_{1}\right\rangle \text { with } \varepsilon_{0}, \varepsilon_{1}>1, \\
E_{K}=H_{K} \times\left\langle\varepsilon_{2}\right\rangle,
\end{gathered}
$$

where $\varepsilon_{2}$ can be chosen as one and only one of the following forms:

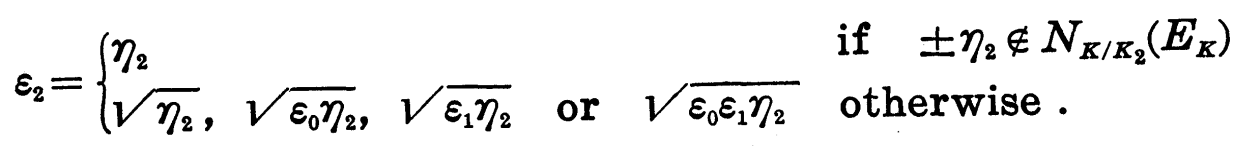

REMARK 2. As in Remark 1, we have

$$
\varepsilon_{2}=\sqrt{\eta_{2}} \text { in }(11) \Longrightarrow N_{K_{2} / e}\left(\eta_{2}\right)=1 \text {. }
$$

1.4. Notations being as in 0.2 , let $n=3$. The fields $K$ and $F$ belong to the case B of 1.2. Note that $E_{K}^{\prime}=E_{K_{2}} \cdot E_{K_{3}}$ and $E_{F}^{\prime}=E_{F_{2}} \cdot E_{K_{3}}$, because $W_{K}=W_{E_{2}}$ and $W_{F}=W_{F_{2}}$.

Assume $L$ is imaginary and the maximal real subfield of $L$ is galois over $\boldsymbol{Q}$, i.e., 


$$
L \cap R=\Omega \text {. }
$$

It is sufficient to consider only $E_{K}$.

Proposition 3. If $n=3$ and $L \cap R=\Omega$, then $E_{\mathbb{R}}=W_{\mathbb{K}_{2}} \cdot E_{\boldsymbol{K}_{8}}$.

Proof. First we mention that $E_{R}^{1-\sigma^{3}}$ is contained in $W_{\mathbb{K}_{2}}$. Because, for $\varepsilon \in E_{\boldsymbol{K}}$ and $i \in \boldsymbol{Z}$,

$$
\left|\varepsilon^{\left(1-\sigma^{8}\right) a^{i}}\right|^{2}=N_{L / \Omega}\left(\varepsilon^{\left(1-\sigma^{3}\right) 0^{i}}\right)=1,
$$

so every conjugate of $\varepsilon^{1-o^{3}}$ has its absolute value 1 , hence $\varepsilon^{1-o^{8}}$ is a root of unity in $K$. Suppose $E_{K} \neq E_{K}^{\prime}$. Then, since $\lambda^{1-o^{3}}=\lambda^{2}$ for $\lambda \in W_{K_{2}}$, there exists $\varepsilon \in E_{K}$ such that

$$
\varepsilon^{o s}=\delta \varepsilon \quad \text { with } \delta= \begin{cases}\sqrt{\overline{-1}} & \text { if } K_{2}=Q(\sqrt{-1}) \\ -1 & \text { otherwise }\end{cases}
$$

on account of Proposition 1 applied as in the case $B$ of 1.2. Now let $K_{2}=Q(\sqrt{-d})$ with a squarefree natural number $d$, and write $\varepsilon=\alpha+\beta V \overline{-d}$ with $\alpha, \beta \in K_{8}$. Then it follows that

$$
\alpha-\beta \sqrt{-d}=\delta(\alpha+\beta \nu \overline{-d}),
$$

which implies that

$$
\varepsilon^{1+\sigma^{3}}= \begin{cases}2 \beta^{2} & \text { if } d=1 \\ d \beta^{2} & \text { otherwise } .\end{cases}
$$

This is a contradiction because the ideal $(2)$ or $(d) \neq(1)$ cannot be a square in $K_{3}$. Hence $E_{K}=E_{K}^{\prime}=E_{K_{3}} \cdot E_{K_{2}}=E_{K_{3}} \cdot W_{K_{2}}$.

Assume $L$ is imaginary and the maximal real subfield of $L$ is not galois over $Q$. Then we may suppose

$$
L \cap R=K \text {. }
$$

In this case, we have

$$
\begin{aligned}
E_{K_{2}}=\langle-1\rangle \times\left\langle\eta_{2}\right\rangle, & E_{K_{3}}=\langle-1\rangle \times\left\langle\eta_{8}\right\rangle \quad \text { with } \\
\left.\eta_{2}, \eta_{3}\right\rangle 1, & E_{F_{2}}=W_{F_{2}}=\langle\rho\rangle .
\end{aligned}
$$

Applying Proposition 1 as in the case $B$ of 1.2, we see

$$
H_{R}=\langle-1\rangle \times\left\langle\varepsilon_{1}\right\rangle \text { with } \varepsilon_{1}>1, \quad H_{F}=W_{F_{2}} \times\left\langle\varepsilon_{0}\right\rangle
$$

and 


$$
E_{K}=H_{K} \times\left\langle\varepsilon_{2}\right\rangle \times\left\langle\varepsilon_{3}\right\rangle, \quad E_{F}=H_{F} \times\left\langle\varepsilon_{3}^{\prime}\right\rangle,
$$

where $\varepsilon_{2}, \varepsilon_{3}$ or $\varepsilon_{3}^{\prime}$ can be taken as one and only one of the following forms:

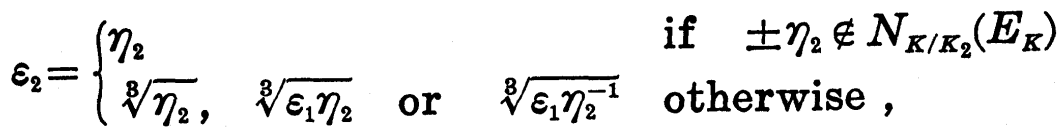

$$
\begin{aligned}
& \varepsilon_{3}=\left\{\begin{array}{lll}
\eta_{8} & & \text { if } \pm \eta_{3} \notin N_{K / K_{3}}\left(E_{K}\right) \\
\sqrt{\eta_{3}} & \text { or } \quad \sqrt{\varepsilon_{1} \eta_{3}} & \text { otherwise ; }
\end{array}\right. \\
& \varepsilon_{3}^{\prime}=\left\{\begin{array}{lll}
\eta_{8} & \text { if } \pm \eta_{8} \notin N_{F / K_{3}}\left(E_{F}\right) \\
\sqrt{\rho \eta_{8}}, \sqrt{\varepsilon_{0} \eta_{3}} & \text { or } \sqrt{\rho \varepsilon_{0} \eta_{3}} & \text { otherwise . }
\end{array}\right.
\end{aligned}
$$

REMARK 3. If $\varepsilon$ is an element of $H_{K}$, we have $N_{K / Q}(\varepsilon)=1$, and so $N_{K / K_{3}}(\varepsilon)=1$, hence

$$
H_{K}=\left\{\varepsilon \in E_{K} \mid N_{K / K_{2}}(\varepsilon)= \pm 1, N_{K / K_{3}}(\varepsilon)=1\right\} \text {. }
$$

Further $N_{K / K_{2}}(\varepsilon)=\operatorname{sgn}(\varepsilon)$ for $\varepsilon \in H_{K}$, therefore

$$
\left\langle\varepsilon_{1}\right\rangle=\left\{\varepsilon \in E_{K} \mid N_{K / K_{2}}(\varepsilon)=N_{K / K_{3}}(\varepsilon)=1\right\} \text {. }
$$

As -1 belongs to $N_{K / K_{2}}\left(E_{K}\right)$, it holds that

$$
\pm \eta_{2} \notin N_{K / K_{2}}\left(E_{K}\right) \Longleftrightarrow \eta_{2} \notin N_{K / K_{2}}\left(E_{K}\right) \text {. }
$$

If $-\eta_{3}$ belongs to $N_{K / K_{3}}\left(E_{K}\right)$, we see that -1 also belongs to $N_{K / K_{3}}\left(E_{K}\right)$ and that

$$
\pm \eta_{3} \notin N_{K / K_{3}}\left(E_{K}\right) \Longleftrightarrow \eta_{3} \notin N_{K / K_{3}}\left(E_{K}\right) \text {. }
$$

Since every element of $N_{F / K_{8}}\left(E_{F}\right)$ is positive, it follows that

$$
H_{F}=\left\{\varepsilon \in E_{F} \mid N_{F / K_{3}}(\varepsilon)=1\right\}
$$

and that

$$
\pm \eta_{3} \notin N_{F / K_{3}}\left(E_{F}\right) \Longleftrightarrow \eta_{3} \notin N_{F / K_{3}}\left(E_{F}\right)
$$

We shall see later in Proposition 6 that $\varepsilon_{3}=\sqrt{\eta_{8}}$ never occurs in (16) and that $\varepsilon_{3}^{\prime}=\sqrt{\rho \eta_{3}}$ never occurs in (17).

Assume $L$ is real, then we may treat only $E_{K}$. We can classify $E_{K}$ similarly be using Proposition 1, though the statements are more complicated. As it is not used in the rest of this paper, we shall omit the explicit formulation of the classification, which is found in Corollary 2. (ii) of [12]. 
1.5. As an example of the case $C$ in 1.2 , we study the following number field. Let $p$ be an odd prime number, $f$ and $g$ be natural numbers such that all $f^{t} g(i=1,2, \cdots, p)$ and $f$ are not perfect $p$-th power in $\boldsymbol{Q}$, and put

$$
k:=Q(\sqrt[p]{f}, \sqrt[p]{g}), \quad k_{i}:=Q\left(\sqrt[p]{f^{i} g}\right)(i=1,2, \cdots, p), k_{p+1}:=Q(\sqrt[p]{f}) .
$$

Then the conditions of $C$ in 1.2 are satisfied with $N=p$ and $M=p+1$, and we have $r_{i}=(p-1) / 2$ as the Dirichlet numbers of $k_{i}(i=1,2, \cdots, p+1)$. On the other hand, the Dirichlet number of $k$ is given by $r=\left(p^{2}-1\right) / 2$. Therefore $r=r_{1}+\cdots+r_{p+1}$ follows. Since there is no proper subfield other than $k_{i}(i=1,2, \cdots, p+1)$, the following proposition is proved by Proposition 1.

Proposition 4. Notations being as above, the group $E_{k} / E_{k}^{\prime}$ is an elementary abelian p-group with p-rank 0 to $\left(p^{2}-1\right) / 2$.

§2. Relations between $E_{K}$ and $E_{F}$.

2.1. Let $n=2$ and keep notations in 0.2 . The following multiplicative homomorphisms are useful to study the relations between $E_{K}$ and $F_{F}$ :

$$
\begin{aligned}
& \phi: K^{\times} \longrightarrow F^{\times} ; x \longmapsto x^{1+o} ; \\
& \psi: F^{\times} \longrightarrow K^{\times} ; y \longmapsto y^{1+\sigma^{8}} .
\end{aligned}
$$

Lemma 1. Let $\phi$ and $\psi$ be as above, then it holds that

$$
\begin{array}{ll}
N_{F / F_{2}}(\phi(x))=N_{K / Q}(x), & N_{K / K_{2}}(\psi(y))=N_{F / Q}(y), \\
\psi \circ \phi(x)=x^{2} N_{K / K_{2}}(x)^{\sigma}, & \phi \circ \psi(y)=y^{2} N_{F / F_{2}}(y)^{\sigma},
\end{array}
$$

for $x \in K^{\times}$and $y \in F^{\times}$.

Proof. Every formula is easily checked by direct calculation.

The next proposition tells us that generators of $E_{K}$ can be utilized to determine generators of $E_{F}$, and vice versa.

Proposition 5. Let $\phi$ and $\psi$ be as above, then we have

$$
\left(H_{K}: W_{K} \cdot \psi\left(H_{F}\right)\right)\left(H_{F}: W_{F} \cdot \phi\left(H_{K}\right)\right)=2 \text { or } 4
$$

respectively when $L \cap R=K$ or $L$ is real. Especially when $L \cap R=K$, it holds that

$$
\boldsymbol{E}_{F}=W_{F} \times\left\langle\phi\left(\varepsilon_{2}\right)\right\rangle, \quad H_{K}=W_{K} \times\left\langle\psi\left(\varepsilon_{0}\right)\right\rangle
$$


if $\varepsilon_{2}=\sqrt{\varepsilon_{1} \eta_{2}}$ in (7). Here $\varepsilon_{0}, \varepsilon_{1}$ and $\eta_{2}$ are given as in (4) and (5).

Proof. By Lemma 1, $\phi$ and $\psi$ induce the homomorphisms

$$
\tilde{\phi}: H_{K} / W_{K} \longrightarrow H_{F} / W_{F} \text { and } \tilde{\psi}: H_{F} / W_{F} \longrightarrow H_{K} / W_{K} \text {, }
$$

which satisfy that $\tilde{\psi} \circ \tilde{\phi}$ and $\tilde{\phi} \circ \tilde{\psi}$ are the squaring endomorphisms of $H_{K} / W_{K}$ and $H_{F} / W_{F}$. Since $H_{K} / W_{K}$ is torsion free, $\tilde{\phi}$ is injective, and so $\tilde{\phi}^{-1}\left(\left(H_{F} / W_{F}\right)^{2}\right)=\tilde{\psi}\left(H_{F} / W_{F}\right)$. Therefore

$$
\left(H_{K} / W_{K}\right) / \tilde{\psi}\left(H_{F} / W_{F}\right) \simeq \tilde{\phi}\left(H_{K} / W_{K}\right) /\left(H_{F} / W_{F}\right)^{2}
$$

Thus

$$
\left(\left(H_{K} / W_{K}\right): \tilde{\psi}\left(H_{F} / W_{F}\right)\right)\left(\left(H_{F} / W_{F}\right): \tilde{\phi}\left(H_{K} / W_{K}\right)\right)=\left(\left(H_{F} / W_{F}\right):\left(H_{F} / W_{F}\right)^{2}\right),
$$

which proves the former part of the proposition on account of (5) and (9). Assume $L \cap \boldsymbol{R}=K$ and $\varepsilon_{2}=\sqrt{\varepsilon_{1} \eta_{2}}$ in (7), then $\phi\left(\varepsilon_{2}\right)$ belongs to $H_{F}$ by Lemma 1 and $\phi\left(\varepsilon_{2}\right)^{2}= \pm \phi\left(\varepsilon_{1}\right)$. This proves the latter by virtue of the former statement.

2.2. Let $n=3$ and keep notations in 0.2 . The following multiplicative homomorphisms are useful to study the relations between $E_{K}$ and $E_{F}$ :

$$
\begin{aligned}
& \phi: K^{\times} \longrightarrow F^{\times} ; \quad x \longrightarrow x^{\sigma+\sigma^{2}} ; \\
& \psi: F^{\times} \longrightarrow K^{\times} ; \quad y \longrightarrow y^{\sigma+\sigma^{2}} .
\end{aligned}
$$

Similarly as in 2.1 , we have

LEMMA 2. Let $\phi$ and \& be as above, then it holds that

$$
\begin{array}{ll}
N_{F / F_{2}}(\phi(x))=N_{K / Q}(x), \quad N_{K / K_{2}}(\psi(y))=N_{F / Q}(y), \\
N_{F / K_{3}}(\phi(x))=N_{K / Q}(x) / N_{K / K_{3}}(x), \quad N_{K / K_{3}}(\psi(y))=N_{F / Q}(y) / N_{F / K_{3}}(y), \\
\psi \circ \phi(x)=x^{-3} N_{K / K_{2}}(x) N_{K / K_{3}}\left(x^{2}\right), \quad \phi \circ \psi(y)=y^{-3} N_{F / F_{2}}(y) N_{F / K_{3}}\left(y^{2}\right),
\end{array}
$$

for $x \in K^{\times}$and $y \in F^{\times}$.

Proof. The formulas are obtained by direct calculation.

Proposition 6. Let $\phi$ and $\psi$ be as above, then we have

$$
\left(H_{K}: W_{K} \cdot \psi\left(H_{F}\right)\right)\left(H_{F}: W_{F} \cdot \phi\left(H_{K}\right)\right)=3 \text { or } 9
$$

respectively when $L \cap R=K$ or $L$ is real. Especially when $L \cap R=K$, it holds that 


$$
H_{F}=W_{F} \times\left\langle\phi\left(\varepsilon_{2}\right)\right\rangle, \quad H_{K}=W_{E} \times\left\langle\psi\left(\varepsilon_{0}\right)\right\rangle
$$

if $\varepsilon_{2}=\sqrt[8]{\varepsilon_{1} \eta_{2}^{ \pm 1}}$ in (15); the case $\varepsilon_{3}=\sqrt{\eta_{8}}$ in (16) or $\varepsilon_{3}^{\prime}=\sqrt{\eta_{8} \rho}$ in (17) never occurs; and the case $\varepsilon_{2}=\eta_{8}$ in (16) occurs if and only if $\varepsilon_{3}^{\prime}=\eta_{3}$ in (17). Here $\rho, \varepsilon_{0}, \varepsilon_{1}, \eta_{2}$ and $\eta_{3}$ are given as in (12) and (13).

Proof. The first statement is proved by Lemma 2 similarly as in the proof of Proposition 5. Let $L \cap R=K$. Assume $\varepsilon_{2}=\sqrt[3]{\varepsilon_{1} \eta_{2}^{ \pm 1}}$ in (15), then $\phi\left(\varepsilon_{2}\right)$ belongs to $H_{F}$ by Lemma 2 and $\phi\left(\varepsilon_{2}\right)^{3}= \pm \phi\left(\varepsilon_{1}\right)$, therefore $H_{F}=$ $W_{F} \times\left\langle\phi\left(\varepsilon_{2}\right)\right\rangle$ and $H_{K}=W_{K} \times\left\langle\psi\left(\varepsilon_{0}\right)\right\rangle$ on account of the first statement of this proposition. Assume $\varepsilon_{8}=\sqrt{\eta_{3}}$ in (16), then $\phi\left(\varepsilon_{2}\right)^{2}=\eta_{3}^{-1}>0$, which is a contradiction because $F \cap R=K_{8}$ and $\phi\left(\varepsilon_{3}\right)$ cannot belong to $K_{8}$. Hence $\sqrt{\eta_{3}}$ does not belong to $K$. Assume $\varepsilon_{3}^{\prime}=\sqrt{\rho \eta_{3}}$ in (17), then $\psi\left(\varepsilon_{3}^{\prime}\right)^{2}=\eta_{3}^{-1}$ which is impossible since $\sqrt{\eta_{8}}$ does not belong to $K$. Hence $\sqrt{\rho \eta_{8}}$ is not an element of $F$. If there is a unit $\varepsilon$ of $K$ such that $N_{K / Z_{3}}(\varepsilon)=\eta_{3}$, see Remark 3, then the third formula of Lemma 2 tells that $N_{F / K_{3}}\left(\phi\left(\varepsilon^{-1}\right)\right)=\eta_{8}$. From the fourth formula of Lemma 2, it is derived that $\eta_{3}$ belongs to $N_{K / K_{3}}\left(E_{K}\right)$ if $\eta_{3}$ is an element of $N_{F / K_{3}}\left(E_{F}\right)$. Thus the proof is complete.

Corollary. When $L \cap R=K$, we have

$$
E_{F}=H_{F} \times\left\langle\phi\left(\varepsilon_{8}\right)\right\rangle, \quad E_{K}=H_{R} \times\left\langle\varepsilon_{2}\right\rangle \times\left\langle\psi\left(\varepsilon_{3}^{\prime}\right)\right\rangle,
$$

where $\varepsilon_{2}, \varepsilon_{3}$ and $\varepsilon_{3}^{\prime}$ are as in (15), (16) and (17). Especially

$$
E_{F}=W_{F} \cdot \phi\left(E_{R}\right)
$$

if $\varepsilon_{2}=\sqrt[3]{\varepsilon_{1} \eta_{2}^{ \pm 1}}$ in (15).

Proof. Combining the first four formulas of Lemma 2 with Proposition 1.(iii), we see the former. Then the latter is clear from Proposition 6.

\section{§3. Minkowski units.}

3.1. Notations being as in 0.2 , we assume $n=2$ and $L \cap R=K$.

Proof of Theorem 2 in CASE $n=2$. Assume $\varepsilon$ is a Minkowski unit for $L$ which belongs to $K$, then

$$
\varepsilon^{1+\sigma^{2}}=N_{L / \Lambda}(\varepsilon)=N_{K / K_{2}}(\varepsilon),
$$

which is not any $\nu$-th power modulo $W_{L}$ in $E_{L}$ for $\nu \geqq 2$. Therefore $\varepsilon^{1+o^{2}}$ is a fundamental unit of both $K_{2}$ and $\Lambda$, is a norm from $E_{K}$ and is not 
a square modulo \pm 1 in $E_{K}$. Hence $E_{A}=W_{A} \times\left\langle\eta_{2}\right\rangle$ and $\varepsilon_{2}=\sqrt{\varepsilon_{1} \eta_{2}}$ in (7). Put $\alpha:=\sqrt{2 \eta_{2}}$ and suppose $K=K_{2}(\alpha)$, then $\phi(\alpha)^{2}=-4$ since $\eta_{2}$ cannot be totally positive, where $\phi$ is given as in 2.1. Therefore $\sqrt{-1}$ is an element of $L$ and $(\alpha /(1-\sqrt{-1}))^{2}=\sqrt{-1} \eta_{2}$. This implies that $\varepsilon^{1+\sigma^{2}}$ is a square modulo $W_{L}$, and a contradiction. Thus $K \neq K_{2}\left(\sqrt{2 \eta_{2}}\right)$. Suppose $K=Q(\sqrt[4]{2})$, then $((1+\omega) / \sqrt[4]{2})^{2}=\omega \eta_{2}$ with $\omega:=(1+\sqrt{-1}) / \sqrt{2}$ and $\eta_{2}=$ $1+\sqrt{2}$. This is also a contradiction. Hence we have proved the "only if" part of the theorem.

Assume $E_{1}=W_{1} \times\left\langle\eta_{2}\right\rangle$ and $\varepsilon_{2}=\sqrt{\varepsilon_{1} \eta_{2}}$, then we see

$$
E_{K}=\left\langle-1, \varepsilon_{2}, \varepsilon_{2}^{\sigma^{2}}\right\rangle \text { and } E_{1}=W_{1} \times\left\langle\varepsilon_{2}^{1+\sigma^{2}}\right\rangle \text {, }
$$

and so it follows from Proposition 5 that

$$
E_{L}^{\prime}=W_{L} \times\left\langle\varepsilon_{2}\right\rangle \times\left\langle\varepsilon_{2}^{o}\right\rangle \times\left\langle\varepsilon_{2}^{\sigma^{2}}\right\rangle .
$$

Therefore, since $E_{L}^{2}$ is contained in $E_{L}^{\prime}$, for every $\xi$ in $E_{L}$,

$$
\xi^{2} \equiv \varepsilon_{2}^{\nu_{0}} \varepsilon_{2}^{\nu_{1}} \sigma \varepsilon_{2}^{\nu_{2} o^{2}} \quad\left(\bmod W_{L}\right)
$$

with $\nu_{i}$ in $Z(i=0,1,2)$. Operating $1+\tau$ and $1+\sigma^{2}$ on the both sides of (19), it is obtained that

$$
\begin{array}{ll}
\xi^{2(1+\tau)} \equiv \varepsilon_{2}^{2 \nu_{2}-\nu_{1}} \varepsilon_{2}^{\left(2 \nu_{2}-\nu_{1}\right) \sigma^{2}} & \left(\bmod W_{K}\right), \\
\xi^{2\left(1+\sigma^{2}\right)} \equiv \varepsilon_{2}^{\left(\nu_{0}+\nu_{2}-\nu_{1}\right)\left(1+\sigma^{2}\right)} & \left(\bmod W_{A}\right) .
\end{array}
$$

So the congruences

$$
\nu_{1} \equiv \nu_{0}+\nu_{2} \equiv 0 \quad(\bmod 2)
$$

are derived. If $\nu_{0} \equiv \nu_{2} \equiv 1(\bmod 2)$, it follows from (19) that there exists a unit $\lambda$ of $L$ such that

$$
\lambda^{2} \equiv \varepsilon_{2}^{1+\sigma^{2}} \equiv \eta_{2} \quad\left(\bmod W_{L}\right) .
$$

If $\lambda^{2}=\eta_{2}$, we see that $\lambda$ is an element of $K=L \cap R$, however it contradicts to $\varepsilon_{2}=\sqrt{\varepsilon_{1} \eta_{2}}$. Therefore we may assume

$$
\lambda^{2}= \begin{cases}-\eta_{2} & \text { if } \sqrt{-1} \notin L \\ \omega \eta_{2} \text { with } \quad \omega:=(1+\sqrt{-1}) / \sqrt{2} & \text { if } \Lambda=Q(\sqrt{2}, \sqrt{-1}) \\ \sqrt{-1} \eta_{2} & \text { otherwise }\end{cases}
$$

When $\sqrt{-1}$ does not belong to $L$, then, since $\lambda$ is neither in $\Lambda$ nor in $K$, we see $\lambda$ is in $K^{\sigma}$ and $\lambda^{\sigma}$ is in $K$, so $\lambda^{2 \sigma}=-\eta_{2}^{o}>0$. Hence $\eta_{2}=-\eta_{2}^{-\sigma}=$ $\left(\lambda^{-\sigma}\right)^{2}$ with $\lambda^{-\sigma}$ in $K$, which also contradicts to $\varepsilon_{2}=\sqrt{\varepsilon_{1} \eta_{2}}$. When $\Lambda=$ 
$Q(\sqrt{2}, \sqrt{-1})$, put $\theta:=(1+\omega) / \lambda$, then $\theta^{2}=\sqrt{2}$ and $K=Q(\theta)=Q(\sqrt[4]{2})$. Otherwise, $\sqrt{-1}$ belongs to $L$ and $\Lambda \neq Q(\sqrt{2}, \sqrt{-1})$, and then $\left(\lambda+\lambda^{\tau}\right)^{2}=$ $2 \eta_{2}$, while $2 \eta_{2}$ is not a square in $K_{2}$ by Satz 13 of [7] since $K_{2} \neq Q(\sqrt{2})$ and $K_{2} \neq Q(\sqrt{3})$. Hence $K=K_{2}\left(\lambda+\lambda^{\tau}\right)=K_{2}\left(\sqrt{2 \eta_{2}}\right)$. Thus we have shown that $\nu_{0} \equiv \nu_{1} \equiv \nu_{2} \equiv 0(\bmod 2)$ on account of $(20)$ if $K \neq Q(\sqrt[4]{2})$ and $K \neq$ $K_{2}\left(\sqrt{2 \eta_{2}}\right)$. So $\xi^{2}$ has already been a square in $E_{L}^{\prime}$ modulo $W_{L}$ by (19), which implies that $E_{L}=E_{L}^{\prime}$ and that, by (18), $\varepsilon_{2}$ is a Minkowski unit for $L$. This completes the proof of Theorem 1 in case $n=2$.

3.2. Notations being as in 0.2 , we assume $n=3$ and $L \cap \boldsymbol{R}=K$.

Proof of Theorem 2 in CASE $n=3$. Assume $\varepsilon$ is a Minkowski unit for $L$ which belongs to $K$, then

$$
\varepsilon^{1+\sigma^{8}}=N_{L / \Omega}(\varepsilon)=N_{K / K_{3}}(\varepsilon),
$$

which is not any $\nu$-th power modulo $W_{L}$ in $E_{L}$ for $\nu \geqq 2$. Therefore $\varepsilon^{1+\sigma^{3}}$ is a fundamental unit of $K_{8}$ and is a norm from $E_{K}$, hence $\varepsilon_{3}=\sqrt{\varepsilon_{1} \eta_{8}}$ in (16) by Proposition 6. Further, since $\varepsilon^{1+a^{8}}$ and $\varepsilon^{\sigma+\sigma^{4}}$ are units of $\Omega$ which, together with $W_{L}$, generate a maximal subgroup with free rank 2 in $E_{L}$, it follows that $E_{\Omega}=W_{\Omega} \times\left\langle\eta_{3}\right\rangle \times\left\langle\eta_{3}^{o}\right\rangle$. Similarly, from

$$
\varepsilon^{1+\sigma^{2}+\sigma^{4}}=N_{L / A}(\varepsilon)=N_{E / K_{2}}(\varepsilon),
$$

we obtain that $E_{1}=W_{1} \times\left\langle\eta_{2}\right\rangle$ and that $\varepsilon_{2}=\sqrt[8]{\varepsilon_{1} \eta_{2}^{ \pm 1}}$ or $\sqrt[8]{\eta_{2}}$. Here $\varepsilon_{2} \neq \sqrt[8]{\eta_{2}}$ because $\varepsilon^{1+\sigma^{2}+\sigma^{4}}$ cannot be a cube in $E_{L}$ modulo $W_{L}$. Thus the "only if" part of the theorem is shown.

Assume $\varepsilon_{2}=\sqrt[8]{\varepsilon_{1} \eta_{2}^{ \pm 1}}$ in (15) and $\varepsilon_{3}=\sqrt{\varepsilon_{1} \eta_{3}}$ in (16), and put $\varepsilon:=\varepsilon_{3} / \varepsilon_{2}$. Then it is easy to see that

$$
E_{K}=W_{K} \times\langle\varepsilon\rangle \times\left\langle\varepsilon^{\sigma^{3}}\right\rangle \times\left\langle\varepsilon^{\sigma^{2}+o^{4}}\right\rangle .
$$

If we further assume $E_{A}=W_{A} \times\left\langle\eta_{2}\right\rangle$ and $E_{\Omega}=W_{\Omega} \times\left\langle\eta_{3}\right\rangle \times\left\langle\eta_{3}^{j}\right\rangle$, then

$$
\begin{aligned}
& E_{\Omega}=W_{\Omega} \times\left\langle\varepsilon^{1+\sigma^{3}}\right\rangle \times\left\langle\varepsilon^{o+\sigma^{4}}\right\rangle, \\
& E_{A}=W_{A} \times\left\langle\varepsilon^{1+\sigma^{2}+\sigma^{4}}\right\rangle .
\end{aligned}
$$

Now, by the corollary of Proposition 6, we see

$$
F_{F}=W_{F} \times\left\langle\varepsilon^{\sigma+\sigma^{2}}\right\rangle \times\left\langle\varepsilon^{\sigma^{4}+\sigma^{5}}\right\rangle .
$$

Therefore

$$
E_{L}^{\prime}=W_{L} \times\langle\varepsilon\rangle \times\left\langle\varepsilon^{0}\right\rangle \times\left\langle\varepsilon^{a^{2}}\right\rangle \times\left\langle\varepsilon^{0^{8}}\right\rangle \times\left\langle\varepsilon^{0^{4}}\right\rangle
$$


and, since $E_{L}^{3}$ is contained in $E_{L}^{\prime}$, for every $\xi$ in $E_{L}$,

$$
\xi^{3} \equiv \varepsilon^{r} \quad\left(\bmod W_{L}\right), \quad \gamma:=\nu_{0}+\nu_{1} \sigma+\cdots+\nu_{4} \sigma^{4}
$$

with $\nu_{i}$ in $Z(i=0,1, \cdots, 4)$. Operating $1+\tau, 1+\sigma^{3} \tau$ and $1+\sigma^{3}$, the congruences

$$
\nu_{i} \equiv 0 \quad(\bmod 3) \quad(i=0,1, \cdots, 4)
$$

are derived similarly as in case $n=2$. Thus $\xi^{8}$ is a cube in $E_{L}^{\prime}$ modulo $W_{L}$, which means $E_{L}=E_{L}^{\prime}$, and hence, by (21), $\varepsilon$ is a Minkowski unit for $L$. This completely proves Theorem 1 in case $n=3$.

3.3. Notations being as in 0.2 , let $n=2$ and $L \cap R=K$. It seems to be a little more complicated to see whether $L$ has a Minkowski unit $\varepsilon$ which is not necessarily real, e.g. $E_{L}=W_{L} \times\langle\varepsilon\rangle \times\left\langle\varepsilon^{0}\right\rangle \times\left\langle\varepsilon^{\tau}\right\rangle$. The following proposition gives a necessary condition for $L$ to have a Minkowski unit.

Proposition 7. Assumptions being as above, let $\eta_{2}$ be as in (4). Then there is no Minkowski unit for $L$ if $\varepsilon_{2}=\eta_{2}$ in (7) and $E_{1} \neq W_{1} \times$ $\left\langle\eta_{2}\right\rangle$.

Proof. Suppose that there is a Minkowski unit $\varepsilon$ for $L$. Then one of the following sets is a system of fundamental units of $L$ :

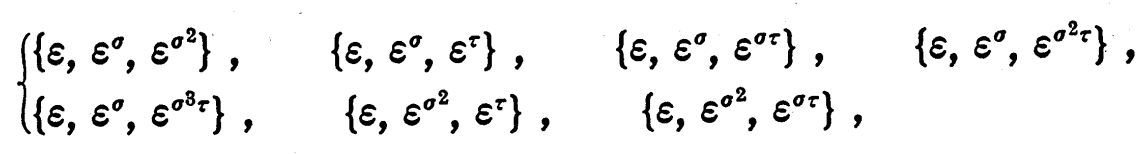

Since $\left\{\varepsilon, \varepsilon^{\sigma}, \varepsilon^{\sigma \tau}\right\}^{\tau}=\left\{\varepsilon^{\sigma \tau},\left(\varepsilon^{\sigma \tau}\right)^{\sigma},\left(\varepsilon^{\sigma \tau}\right)^{\tau}\right\},\left\{\varepsilon, \varepsilon^{\sigma}, \varepsilon^{\sigma^{2} \tau}\right\}^{\sigma}=\left\{\varepsilon^{\sigma},\left(\varepsilon^{\sigma}\right)^{\sigma},\left(\varepsilon^{\sigma}\right)^{\tau}\right\}$ and $\left\{\varepsilon, \varepsilon^{\sigma}, \varepsilon^{\sigma \tau} \tau\right\}^{\sigma \tau}=$ $\left\{\varepsilon^{\sigma^{2} \tau},\left(\varepsilon^{a^{2} \tau}\right)^{\sigma},\left(\varepsilon^{\sigma^{2} \tau}\right)^{\tau}\right\}$, we may treat the first two and the last two sets of (22). If the first set of (22) is a system of fundamental units of $L$, we have

$$
E_{A}=W_{A} \times\left\langle\varepsilon^{1+\sigma^{2}}\right\rangle \text {. }
$$

If $\varepsilon^{1+\sigma^{2}}$ is not a fundamental unit of $K_{2}$, i.e., $E_{A} \neq W_{1} \times\left\langle\eta_{2}\right\rangle$, then $\varepsilon^{\left(1+\sigma^{2}\right)(1+\tau)}$ is a fundamental unit of $K_{2}$. From

$$
\varepsilon^{\left(1+\sigma^{2}\right)(1+\tau)}=\varepsilon^{(1+\tau)\left(1+\sigma^{2}\right)},
$$

it follows that $\eta_{2}$ is element of $N_{K / K_{2}}\left(E_{K}\right)$ and that $\varepsilon_{2} \neq \eta_{2}$ in (7). Therefore $E_{1}=W_{1} \times\left\langle\eta_{2}\right\rangle$ or $\varepsilon_{2} \neq \eta_{2}$ in (7) if the first set of (22) is a system of fundamental units of $L$. Similarly, if either of the last two sets of (22) is a system of fundamental units of $L$, we have $E_{1}=W_{A} \times\left\langle\eta_{2}\right\rangle$ or $\varepsilon_{2} \neq \eta_{2}$ 
in (7). If the second set of (22) is a system of fundamental units of $L$, it holds that

$$
\varepsilon^{\sigma \tau} \equiv \varepsilon^{\nu} \varepsilon^{\nu_{1} \sigma} \varepsilon^{\nu_{2} \tau} \quad\left(\bmod W_{L}\right)
$$

with $\nu_{i}$ in $Z(i=0,1,2)$. Then, operating $\tau$ on the both sides, we obtain that

$$
\varepsilon^{\sigma} \equiv \varepsilon^{\nu_{0} \nu_{1}+\nu_{2}} \varepsilon_{1}^{\nu_{1}^{2} \sigma} \varepsilon^{\left(\nu_{0}+\nu_{1} \nu_{2}\right) \tau} \quad\left(\bmod W_{L}\right)
$$

and that

$$
\varepsilon^{\sigma \tau} \equiv \varepsilon^{\nu_{0}} \varepsilon^{\nu_{1} \sigma} \varepsilon^{-\nu_{1} \nu_{0} \tau} \quad\left(\bmod W_{L}\right)
$$

with $\nu_{1}= \pm 1$. Therefore

$$
\varepsilon^{o(1+\tau)} \equiv \varepsilon^{\nu_{0}\left(1-\nu_{1} \tau\right)} \varepsilon^{\left(1+\nu_{1}\right) \sigma} \quad\left(\bmod W_{L}\right) .
$$

Since $N_{L / K}\left(E_{L}\right)$ is a finite index subgroup of $E_{K}, \varepsilon^{1+\tau}$ and $\varepsilon^{o(1+\tau)}$ are independent units of $K$, so $\nu_{1}=1$ and

$$
\varepsilon^{o \tau} \equiv \varepsilon^{\nu_{0}} \varepsilon^{\sigma} \varepsilon^{-\nu_{0} \tau} \quad\left(\bmod W_{L}\right) .
$$

Now, for $\xi$ in $E_{K}$, let

$$
\xi \equiv \varepsilon^{\mu_{0}} \varepsilon^{\mu_{1} \sigma} \varepsilon^{\mu_{2} \tau} \quad\left(\bmod W_{L}\right)
$$

with $\mu_{i}$ in $Z(i=0,1,2)$, then, on account of $\xi=\xi^{\tau}$, it follows from (23) that

$$
\xi \equiv \varepsilon^{\mu_{0}(1+\tau)} \varepsilon^{\mu_{1}\left(\sigma-\nu_{0} \tau\right)} \quad\left(\bmod W_{L}\right) .
$$

If $E_{1} \neq W_{1} \times\left\langle\eta_{2}\right\rangle$, then $\eta_{2}$ is a square modulo $W_{A}$ in $E_{A}$ by Satz 12 of [7]. Therefore, when $\xi=\eta_{2}$ in (24), all the $\mu_{i}(i=0,1,2)$ should be even, hence, by (25), $\eta_{2}$ is a square modulo $W_{L}$ in $E_{K} \cdot W_{L}$. Thus, by $E_{K}$. $W_{L} / W_{L} \simeq E_{K} / W_{K}, \eta_{2}$ is a square modulo $W_{K}$ in $E_{K}$, i.e. $\varepsilon_{2}=\sqrt{\eta_{2}}$ in (7), and the proof is complete.

\section{§4. Binomial units.}

4.1. For $n=2$ or 3 , let $K=Q(\theta), \theta=\sqrt[2 n]{d}$ with $2 n$-th power free $d$ in $N$, be a real pure number field of degree $2 n$, and $\zeta$ be a primitive $2 n$-th root of unity. Then the galois closure $L$ of $K / Q$ and the galois group $G$ of $L / Q$ are given by

$$
L=Q(\theta, \zeta)=K(\zeta) ; \quad G=\langle\sigma, \tau\rangle, \quad \theta^{\sigma}=\zeta \theta, \quad \theta^{\tau}=\theta, \quad \zeta^{\sigma}=\zeta, \quad \zeta^{\tau}=\zeta^{-1} .
$$


The group $G$ is a dihedral group of order $4 n$. Using the notations in 0.2 , we see that

$$
L \cap R=K, \quad F=Q\left(\sqrt[2 n]{-n^{n} d}\right) .
$$

We determine fundamental units of $K, F$ and $L$ explicitly in a certain case, assuming that $K$ has a binomial unit. Before we prove Theorems 2 and 3, we make a few remarks.

REMARK 4. Let $S$ be the set of $K$ which has a unit of type $a-b \theta$ with $a, b$ in $Z, a>0, b>1$. Then, by Ljunggren's theorem (Satz 3 and Satz 7 of [17]), there is only one unit of type $a-b \theta$ in $K$ for each $K$ in $S$, and the fields $K$ differ if the pairs $(a, b)$ differ. Therefore, if we put

$$
S_{b}:=\left\{K \in S \mid a \in N, a-b \theta \in E_{K}\right\}
$$

for each $b>1$, we see

$$
S=\bigcup_{b=2}^{\infty} S_{b} \quad \text { (disjoint union). }
$$

So we fix $b>1$ and consider $K$ it $S_{b}$. Then, by a similar manner as in H. Yokoi [18], we see that $a-b \theta$ is a unit of $K$ if and only if

$$
\begin{aligned}
& a=b^{2 n} c+a_{0} \\
& d= \begin{cases}c\left(b^{4} c+2 a_{0}\right)\left(b^{8} c^{2}+2 a_{0} b^{4} c+2 a_{0}^{2}\right)+d_{0} & \text { for } n=2 \\
c\left(b^{8} c+2 a_{0}\right)\left(b^{12} c^{2}+a_{0} b^{6} c+a_{0}^{2}\right)\left(b^{12} c^{2}+3 a_{0} b^{8} c+3 a_{0}^{2}\right)+d_{0} & \text { for } n=3\end{cases}
\end{aligned}
$$

with a certain natural number $c$ and a rational integer $a_{0}$ such that

$$
a_{0}^{2 n} \equiv \pm 1\left(\bmod b^{2 n}\right), \quad \frac{1 \pm 1}{2}-b^{2 n}<a_{0} \leqq \frac{1 \pm 1}{2} .
$$

Here the rational integer $d_{0}$ is given by $a_{0}^{2 n}= \pm 1+b^{2 n} d_{0}$. If $c>1$, we see $a>b^{2 n}$, so the assumption $a \geqq b^{2 n}-1$ removes only finitely many fields in $S_{b}$. Especially in the trivial case in (26), $a_{0}= \pm 1$,

$$
d= \begin{cases}c\left(b^{4} c \pm 2\right)\left(b^{8} c^{2} \pm 2 b^{4} c+2\right) & \text { for } n=2, \\ c\left(b^{6} c \pm 2\right)\left(b^{12} c^{2} \pm b^{8} c+1\right)\left(b^{12} c^{2} \pm 3 b^{8} c+3\right) & \text { for } n=3 .\end{cases}
$$

By [9], we can prove, for any fixed $b$ in $N$, odd in case $n=2$, there are infinitely many square free $d$ of the form (27). Therefore there are infinitely many fields in the set $S_{b}$ (b: odd if $n=2$ ) which satisfy the assumption of Theorem 2 . In the case $d$ is as in (27), the condition 
$a \geqq b^{2 n}-1$ is satisfied even if $c=1$. The same fact holds for $S_{b}$ when $b$ is even and $n=2$.

We note that $d$ becomes very large since $d$ is a polynomial of degree $2 n(2 n-1)$ in $b$ and of degree $2 n$ in $c$.

Remark 5. By using Satz 3, Satz 7, Satz 16 and Satz 22 of [17], we see that the field $K$ in Theorem 2 is different from those in Stender [17] unless $b=1$. Of course, Theorem 3 is a new result including the case $b=1$.

4.2.

Proof of Theorem 2. (i) Let $n=2$. When $b=1$, the proof is seen in [15]. Assume $b>1$. Then the inequalities

$$
a \geqq 15, \quad \operatorname{Max}(a, b \theta)<a+1, \quad \theta^{-1}<\sqrt[4]{a+1} /(a-1)
$$

are obtained easily. Let $\delta:=a^{4}-b^{4} d=N_{E / Q}\left(\xi_{1}\right)$, and $\eta_{2}, \varepsilon_{1}$ be as in (4), (5). Then

$$
\eta_{2}=\delta \xi_{1}^{-1} \xi_{2}^{-1}=\delta N_{K / K_{2}}\left(\xi_{2}^{-1}\right)=a^{2}+b^{2} \theta^{2}
$$

and

$$
\varepsilon_{1}=\xi_{2}^{2} \eta_{2}=\delta \xi_{1}^{-1} \xi_{2}=\left(a^{2}+b^{2} \theta^{2}\right)(a+b \theta)^{2}
$$

are proved as follows. In (29), only the first equality is non-trivial. Suppose $a^{2}+b^{2} \theta^{2}=\eta_{2}^{\nu}$ with $\nu \geqq 2$, then

$$
1 \leqq \theta^{-2}\left(\eta_{2}+\left|\eta_{2}^{o}\right|\right)<\theta^{-2}\left(\sqrt{a^{2}+b^{2} \theta^{2}}+1\right)
$$

since $0 \neq \theta^{-2}\left(\eta_{2}-\eta_{2}^{o}\right) \in Z$ and $1<\eta_{2} \leqq \sqrt{a^{2}+b^{2} \theta^{2}}$. On the other hand

$$
\theta^{-2}\left(\sqrt{a^{2}+b^{2} \theta^{2}}+1\right)<1
$$

follows from (28). This is a contradiction, and so (29) is proved. In (30), only the first equality is non-trivial. Note that $\sqrt{\eta_{2}}$ does not belong to $K$ because, if it belongs to $K$, we have $\phi\left(\sqrt{\eta_{2}}\right)^{2}=-1$, though $F_{2} \neq Q(\sqrt{-1})$, where $\phi$ is given as in 2.1. Suppose $\xi_{2}^{2} \eta_{2}=\varepsilon_{1}^{\nu}$ with $\nu \geqq 3$. Then, by (3), (4), we see $1<\theta^{-8}\left(3+\sqrt[3]{\xi_{2}^{2} \eta_{2}}\right)$, applying Hilfssatz 1 of [15]. While $\theta^{-8}\left(3+\sqrt[8]{\xi_{2}^{2} \eta_{2}}\right)<1$ follows from (28). This is a contradiction, and hence (30) is proved. Thus, by (29), (30), and by (6), (7), Theorem 2 is completely proved in case $n=2$.

(ii) Let $n=3$. When $b=1$, the proof is seen in [16]. So we assume $b>1$. Then the inequalities 


$$
a \geqq 63, \quad \operatorname{Max}(a, b \theta)<a+1, \quad \theta^{-1}<\sqrt[6]{a+1} /(a-1)
$$

are proved easily. Similarly as in case $n=2$, we obtain

$$
\begin{aligned}
& \eta_{2}=\delta \xi_{1}^{-1} \xi_{3}^{-1}=\delta N_{K / K_{2}}\left(\xi_{1}^{-1}\right)=a^{8}+b^{8} \theta^{3}, \\
& \eta_{3}=\delta \xi_{1}^{-1} \xi_{2}^{-1}=\delta N_{K / K_{8}}\left(\xi_{1}^{-1}\right)=a^{4}+a^{2} b^{2} \theta^{2}+b^{4} \theta^{4}, \\
& \varepsilon_{1}=\xi_{1}^{-6} \eta_{2}^{-2} \eta_{3}^{-8}=\delta \xi_{1}^{-1} \xi_{2}^{8} \xi_{3}^{2}=(a+b \theta)^{8}\left(a^{2}+a b \theta+b^{2} \theta^{2}\right)^{2}\left(a^{5}+a^{4} b \theta+\cdots+b^{5} \theta^{5}\right)
\end{aligned}
$$

by using (31) and the results in [16]. Here $\delta=a^{6}-b^{6} d=N_{E / Q}\left(\xi_{1}\right)$, and $\varepsilon_{1}$, $\eta_{2}$ and $\eta_{8}$ are given as in (12) and (13). The detailed proofs of (32), (33) and (34) are done in the same way as in [11], so they are omitted. Now we see, on account of (32), (33), (34), that $\varepsilon_{2}=\sqrt[8]{\varepsilon_{1} \eta_{2}^{-1}}=\xi_{2} \xi_{3}$ in (15), $\varepsilon_{8}=$ $\sqrt{\varepsilon_{1} \eta_{3}}=\delta \xi_{1}^{-1} \xi_{2} \xi_{8}$ in (16), and that $\xi_{1}, \xi_{2}, \xi_{8}$ form a set of fundamental units of $K$. Thus the proof of Theorem 2 is complete in case $n=3$.

4.3.

PrOOF OF THEOREM 3. (i) Let $n=2$. Then $K \neq K_{2}\left(\sqrt{2 \eta_{2}}\right)$, because $F_{2} \neq Q(\sqrt{-1})$. Therefore, on account of Theorem 1 , it is sufficient to show

$$
E_{\Lambda}=W_{A} \times\left\langle\eta_{2}\right\rangle \text {, }
$$

since $\sqrt{\varepsilon_{1} \eta_{2}}=\delta \xi_{1}^{-1} \in K$ has already been shown in the proof of Theorem 2. We mention that $d>3$ by (29) and by the assumption. Then we can apply Satz 13 of [7] to prove (35). Assume that the ideal (2) is a square of a principal ideal in $K_{2}$. Then $2 \eta_{2}=\left(\left(x+y \theta^{2}\right) / 2\right)^{2}$ with $x, y$ in $Z, x \equiv y$ $(\bmod 2)$. From this and $(29), 4 b^{2}=x y$ and $x \equiv y \equiv 0(\bmod 2)$ follow. Therefore

$$
2 \eta_{2}=\left(s+t \theta^{2}\right)^{2}
$$

with $s, t$ in $Z$. Taking the norms of the both sides, we have $4=\left(s^{2}-t^{2} d\right)^{2}$. Hence

$$
2 a^{2}=s^{2}+t^{2} d, \quad \pm 2=s^{2}-t^{2} d,
$$

and so $a^{2} \pm 1=8^{2}$. This is not the case and (35) follows from Satz 13 of [7] since $d>3$. The proof is complete in case $n=2$.

(ii) Let $n=3$. It is sufficient to show

$$
E_{1}=W_{1} \times\left\langle\eta_{2}\right\rangle \text { and } E_{\Omega}=W_{\Omega} \times\left\langle\eta_{8}\right\rangle \times\left\langle\eta_{3}^{o}\right\rangle
$$

on account of Theorem 1 , because $\varepsilon_{2}=\sqrt[8]{\varepsilon_{1} \eta_{2}^{-1}}$ in (15), $\varepsilon_{3}=\sqrt{\varepsilon_{1} \eta_{3}}$ in (16) 
and $\varepsilon_{3} / \varepsilon_{2}=\delta / \xi_{1}$ have been shown in the proof of Theorem 2. Mention that $d>3$ by (32) and by the assumption. Assume $E_{A} \neq W_{A} \times\left\langle\eta_{2}\right\rangle$, then, applying Satz 14 of [7] similarly as in the proof of Proposition 4.(i) of [11], we have

$$
3 a^{3}=x^{2}+y^{2} d, \quad \pm 3=x^{2}-y^{2} d, \quad 3 b^{3}=2 x y,
$$

with $x, y$ in $Z$. From this, it follows that $3 \mid x,(x / 3, y)=1$ and that $x=3 z^{3}$ or $x=12 z^{3}$ with $z$ in $Z$. Therefore

$$
a^{3} \pm 1=6\left(z^{2}\right)^{3} \text { or } 12\left(2 z^{2}\right)^{3}
$$

with $z$ in $Z$, which is impossible, hence $E_{A}=W_{A} \times\left\langle\eta_{2}\right\rangle$. Assume $E_{Q} \neq$ $W_{s} \times\left\langle\eta_{3}\right\rangle \times\left\langle\eta_{3}^{o}\right\rangle$, then, applying Proposition 1.2 of [6] similarly as in the proof of Proposition 4.(ii) of [11], we have

$$
3\left(a^{4}+\delta a^{2}+1\right)=(x+y)^{2}-3 x y
$$

with $x, y$ in $Z$ such that $a^{4}+\delta a^{2}+1$ divides $b^{4}(x+y)$. Note here that $b^{8} d=\left(a^{2}-\delta\right)\left(a^{4}+\delta a^{2}+1\right),\left(a^{2}-\delta\right)^{2}+3 \delta a^{2}=a^{4}+\delta a^{2}+1$ and that $d$ is square-free. Therefore every prime divisor of $a^{4}+\delta a^{2}+1$ divides $b$, hence

$$
a^{4}+\delta a^{2}+1 \leqq b^{7} \text {. }
$$

On the other hand, we have

$$
a^{4}+\delta a^{2}+1 \geqq\left(b^{6}-1\right)^{4}-\left(b^{6}-1\right)^{2}+1 .
$$

This is impossible except for $b=1, \delta=-1$ and $a=1$, and then $d=2$, which is the case removed by the assumption. Therefore $E_{\Omega}=W_{0} \times\left\langle\eta_{3}\right\rangle \times\left\langle\eta_{3}^{o}\right\rangle$. Thus (36) is shown and the proof is complete in case $n=3$.

4.4. Lastly, we give an example of $L$ which has no Minkowski unit, real or not, in case $n=2$.

Proposition 8. The field $L=Q\left(\sqrt[4]{3 g^{2}}, \sqrt{-1}\right)$ has no Minkowski unit if $g$ is a square free natural number prime to 3.

Proof. We apply Proposition 7. Notations being as in 0.2 , we see $\Lambda=Q(\sqrt{3}, \sqrt{-1})$. Let $\eta_{2}$ be as in (4), then $\eta_{2}=2+\sqrt{3}$ and

$$
E_{1} \neq W_{1} \times\left\langle\eta_{2}\right\rangle \text {. }
$$

Put $\theta:=\sqrt[4]{3 g^{2}}$, then, for $\varepsilon$ in $E_{K}$, it follows from [15] that

$$
2 g \varepsilon \theta=g x_{0}+g x_{1} \theta+x_{2} \theta^{2}+x_{8} \theta^{8}
$$


with rational integers $x_{i}(i=0,1,2,3)$. If $N_{K / K_{2}}(\varepsilon)=\eta_{2}=2+\sqrt{\mathbf{3}}$,

$$
\left\{\begin{array}{l}
x_{0}^{2}+3 x_{2}^{2}-6 g x_{1} x_{3}=-12 g \\
2 x_{0} x_{2}-g x_{1}^{2}-3 g x_{3}^{2}=-8 g
\end{array}\right.
$$

are derived. But this is impossible, hence $\varepsilon_{2}=\eta_{2}$ in (7), see also Remark 1. Therefore the assumptions of Proposition 7 are satisfied, and $L$ has no Minkowski unit.

\section{References}

[1] K. K. Billevich, A theorem on unit elements of algebraic fields of order $n$, Mat. Sb., N.S., 64 (106) (1964), 145-152. (Russian) MR 29, 1201.

[2] Z. I. Borevich and I. R. Shafarevich, Number Theory, Academic Press, New YorkLondon, 1966.

[ 3 ] L. Bouvier and J.-J. Payan, Modules sur certains anneaux de Dedekind, J. Reine Angew. Math., 274/275 (1975), 278-286.

[4] A. BRUMer, On the group of units of an absolutely cyclic number field of prime degree, J. Math. Soc. Japan, 21 (1969), 357-358.

[5] G. Gras and M.-N. Gras, Calcul du nombre de classes et des unités des extension abéliennes réelles de $Q$, Bull. Soc. math. France, $2^{e}$ série, 101 (1977), 97-129.

[6] K. Imura, On the unit groups of certain sextic number fields, Abh. Math. Sem. Univ. Hamburg, 50 (1980), 32-39.

[ 7 ] S. KuRodA, Uber den Dirichletschen Körper, J. Fac. Sci. Univ. Tokyo, Sect. IA, 4 (1943), 383-406.

[8] N. Moser, Unités et nombre de classes d'une extension Galoisienne diédrale de $\boldsymbol{Q}, \mathrm{Abh}$. Math. Sem. Univ. Hamburg, 48 (1979), 54-75.

[9] T. Nageld, Zur Arithmetik der Polynome, Abh. Math. Sem. Univ. Hamburg, 1 (1922), 174-194.

[10] T. NAGELL, Sur quelques question dans la théorie des corps biquadratiques, Ark. Mat., 4 (1961), 347-376.

[11] K. NARAmula, An explicit formula for the fundamental units of a real pure sextic number field and its galois closure, Pacific J. Math., 83 (1979), 463-471.

[12] K. Nakamula, On the group of units of a non-galois quartic or sextic number field, Proc. Japan Acad., Ser. A, 56 (1980), 77-81.

[13] K. Naramula, Class number calculation and elliptic unit, II Quartic case, Proc. Japan Acad., Ser. A, 57 (1981), 117-120.

[14] K. Nakamula, Class number calculation and elliptic unit, III Sextic case, Proc. Japan Acad., Ser. A, 57 (1981), 363-366.

[15] H.-J. STENDER, Grundeinheiten für einige unendlich Klassen biquadratischen Zahlkörper mit einer Anwendung auf die diophantische Gleichung $x^{4}-a y^{4}= \pm c(c=1,2,4$ oder 8), J. Reine Angew. Math., 264 (1973), 207-220.

[16] H.-J. STENDER, Uber die Einheitengruppe der reinen algebraischen Zahlkörper sechsten Grades, J. Reine Angew. Math., 268/269 (1974), 78-93.

[17] H.-J. STENDER, Lösbare Gleichungen $a x^{n}-b y^{n}=c$ und Grundeinheiten für einige algebraische Zahlkörper vom Grade $n, n=3,4,6$, J. Reine. Angew. Math., 290 (1977), 24-62.

[18] H. YokoI, The diophantine equation $x^{3}+d y^{8}=1$ and the fundamental unit of a real pure 
cubic field $Q(\sqrt[8]{d})$, J. Reine Angew. Math., 268/269 (1974). 174-179.

Present Address: Department of Mathimatics FACULTY OF SCIENCES TORYO METROPOLITAN UNIVERSITY Furazawa, Setagaya-ro, Tozyo, 158 